\title{
Diversification du vivant sans modification du génome : enjeux didactiques et épistémologiques
}

\author{
Corinne Fortin \\ École Supérieure du Professorat et de l'Éducation- Université Paris-Est Créteil (ESPE-UPEC). Rue \\ Jean Macé, 94380 Bonneuil-sur-Marne. France
}

\begin{abstract}
Résumé. Le programme français de la classe de Terminale Scientifique évoque dans un de ces items la diversification des êtres vivants sans modification du génome. En nous appuyant sur les prescriptions officielles, nous analysons des implications didactiques et épistémologiques liées à l'introduction de l'hérédité non génétique ou l'héritabilité des comportements comme nouveau référent paradigmatique au regard de la vision géno-centrique dominante de la diversification du vivant. Plus précisément, nous nous intéressons à la nature de la biologie évolutive qui est donnée à voir aux élèves dans le cadre de concepts émergents. Au terme de l'analyse, il apparaît que le programme privilégie une approche descriptive de la diversification du vivant sans faire appel, explicitement, à un substrat théorique.
\end{abstract}

\begin{abstract}
The diversification of living beings without modification of the genome: didactical and epistemological issue. The French curriculum of Scientific Terminal Level has introduced a new item concerning the Diversification of living beings without modification of the genome By drawing on the official requirements about this item, we analyze didactic and epistemological implications of the introduction of a new paradigmatic referents such as non-hereditary genetic or hereditability of behavior compared to the dominant geno-centric focus about the diversification of living world. Specifically, we are interested by the nature of evolutionary biology that is given to see students as part of the emergence of a new concepts After the analysis, it appears that the curricula emphasizes a descriptive approach to the diversification of living world without calling explicitly to a theoretical substrate.
\end{abstract}

\section{Introduction}

Le programme français de Sciences de la vie et de la Terre (SVT), en classe de Terminale Scientifique (T.S), évoque la diversification des êtres vivants avec et sans modification du génome. Les précédents programmes de la classe de TS ne faisaient pas fait référence à la diversification du vivant sans modification du génome. Certes, il était fait mention d'une possible accommodation, mais dans le cadre d'une réponse adaptative à un changement environnemental, et non pas comme pouvant être à l'origine de la diversification du vivant. Cette partie du programme fait écho au célèbre ouvrage d'Henri Atlan (1999) [1] La fin du tout génétique ? Vers de nouveaux paradigmes en biologie, car il s'agit de reconsidérer la centralité explicative du rôle des gènes dans la construction, la diversification, et l'évolution du vivant. Elle fait aussi écho au concept d'hérédité non génétique, 
c'est-à-dire une transmission, de génération en génération, d'informations non codées dans l'ADN. Parmi les formes d'hérédité non génétique, Danchin et al. (2011) [2] distingue l'hérité épigénétique quand la variation de l'expression des gènes, en fonction de l'environnement, est transmise à la descendance ; l'hérédité écologique liée au fait que les descendants héritent de l'habitat de leur parents ; l'hérédité par effet parentaux quand l'action des parents influe sur le phénotype des descendants, et l'hérédité culturelle liée à la transmission sociale de certains comportements appris. Pour expliquer l'importance des mécanismes non génétiques, certains chercheurs proposent une extension de théorie actuelle de l'évolution (Pigliucci et Müller, 2010) [3]. Cette extension, selon eux, vise à dépasser la vision "géocentrique ${ }^{1}$ » ou vision selon laquelle les mécanismes évolutifs seraient exclusivement d'ordre génétique. L'extension de la théorie n'est donc pas seulement l'intégration de nouvelles données sur l'épigenèse, la plasticité phénotypique, etc. dans le schème explicatif traditionnel de la théorie de l'évolution. Il s'agit, en réalité, de le dépasser pour reconnaître dans l'épigenèse, la plasticité, etc. non pas des productions consécutives à l'action de mécanismes évolutifs génétiques, mais bien des processus non génétiques ayant un rôle moteur dans l'évolution. Ce point de vue est loin d'être partagé par l'ensemble de la communauté scientifique et fait l'objet de vifs débats entre les partisans du maintien et ceux de l'extension de la théorie de l'évolution. L'illustration ci-dessous situe les principaux concepts de la théorie dite extensive (ou inclusive) au regard de ceux de l'actuelle théorie de l'évolution.

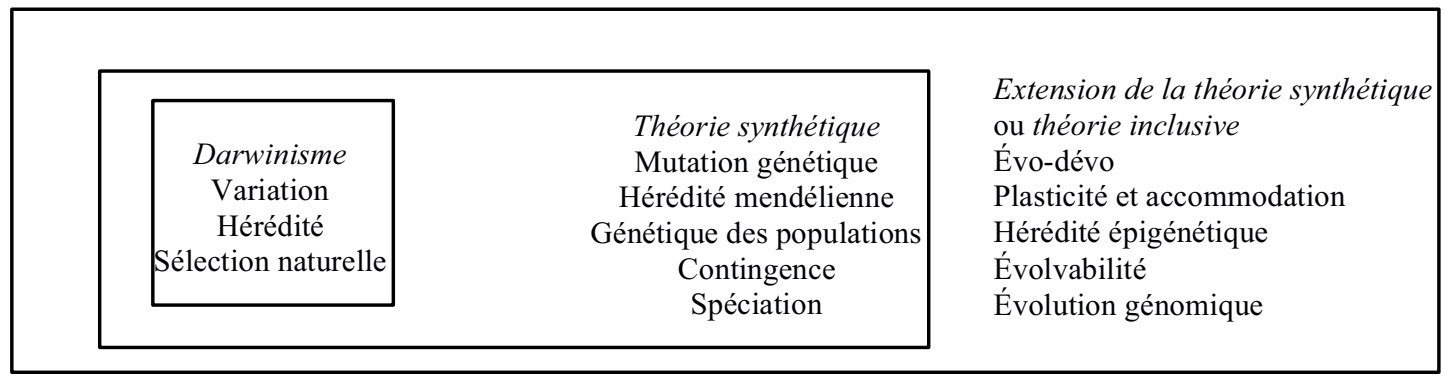

Figure 1. Représentation des concepts clés des différents champs théoriques (d'après Pigliucci et Müller, 2010) [3]

Cette représentation inclusive ne traduit pas une rupture ou un changement paradigmatique avec l'actuelle théorie de l'évolution. Néanmoins, l'inclusion délimite une frontière pour chacun des sousensembles, et traduit ainsi une forme d'irréductibilité liée à un changement de référent paradigmatique. Changement de référent au sens où l'extension de la théorie n'accorde plus un poids prédominant, voire exclusif, à la mutation génétique et à la sélection naturelle, mais décentre ses explications sur des processus causaux non génétiques d'évolution. Dans le programme, la diversification non génétique des êtres vivants se situe à la marge puisque l'essentiel porte sur la diversification d'origine génétique (ex : mutation, recombinaison génétique, duplication génétique, etc..). Cependant, se pose la question de la « Nature de la Science» enseignée $\left(\mathrm{NoS}^{2}\right)$ au sens de Lederman (1992) [4]. S'agit-il d'une science stabilisée dans le cadre de théorie actuelle de l'évolution ou bien une science en devenir avec l'émergence de la théorie extensive de l'évolution ? Dans cet article, nous interrogerons la place donnée à diversification du vivant sans modification du génome, dans une perspective à la fois didactique et épistémologique, comme un prolongement, un renouvellement ou une rupture avec l'actuelle théorie de l'évolution.

Le présent article ne relève pas d'une recherche à proprement parler. C'est plutôt une lecture didactique (avec sa part de subjectivité) de la dimension épistémologique d'un nouvel item. Dans un premier temps, nous présenterons le cadre conceptuel relatif à la distinction entre causes proximales et distales de la diversification du vivant en lien avec la caractérisation de la nature de la causalité évolutive comme une composante de la nature de la biologie de l'évolution. Dans un deuxième

\footnotetext{
${ }^{1}$ Danchin (2011) [2] dans un entretien au Monde du 31/01/2012 précise « Aujourd'hui, quand on pense évolution.

${ }^{2} \mathrm{NoS}$ : Acronyme de Nature of Science.
} 
temps, nous proposerons une analyse de l'item consacré à la diversification du vivant sans modification du génome en nous appuyant d'une part, sur les prescriptions du Programme officiel de l'Éducation Nationale de SVT en classe de T.S, et d'autre part, sur la publication par l'Inspection Générale de l'Éducation Nationale de Sciences de la vie et de la Terre (IGEN SVT) de Ressources pour la classe de Terminale Scientifique.

En conclusion, nous discuterons de l'apport de cette analyse dans la perspective d'un enseignement de la nature de la biologie de l'évolution incluant la dimension génétique et non génétique de la diversification du vivant.

\section{Cause proximales et distales de la diversification du vivant : une dichotomie discutée}

Dans un article devenu une référence épistémologique, Mayr énonçait en 1961 [5] la distinction entre causes proximales agissant sur le fonctionnement des organismes et causes distales relatives à la formation des espèces sous l'action de la sélection naturelle. Il distinguait ainsi la biologie fonctionnaliste de la biologie historique ou évolutive. Les explications fonctionnalistes font appel à des causales proximales à fort pouvoir prédictif proche du déterminisme physiologique ou des probabilités en génétique des populations. Tandis que les explications évolutionnistes font appel à des causales distales à faible pouvoir prédictif puisqu'il s'agit d'expliquer, rétroactivement, la production d'événements singuliers et imprévisibles par l'action de mécanismes biologiques non dirigés (exemple: mutation, la sélection naturelle ou la dérive génétique). Cette dichotomie explicative est aujourd'hui nuancée, voire rediscutée, à la lumière des recherches sur les processus évolutifs non génétiques. Des travaux menés par Agrawal (2001) [6] ces dernières années sur la plasticité phénotypique, c'est-à-dire sur la possibilité qu'un même génotype puisse donner différents phénotypes en fonction des variations de l'environnement, ont montré que la sélection naturelle peut maintenir certains états plastiques ayant une fitness (valeur sélective) supérieure à celle des individus non plastiques. Traditionnellement étudiée sous l'angle des causes proximales d'une adaptation provisoire aux changements de milieu par accommodation, comme étant le produit d'une induction immédiate du milieu sur le phénotype, la plasticité phénotypique est désormais aussi étudiée sous l'angle des causes distales (Piersmai et Van Gils, 2011) [7]. Dans le cas où la plasticité phénotypique est sujette à sélection, causes proximales et distales sont alors étroitement connectées et se situent à la croisée de l'action de l'environnement, du développement de l'organisme et de l'histoire de l'espèce. L'explication causale de cette plasticité réduit significativement l'écart entre biologie fonctionnaliste et biologie historique. Il apparaît alors de plus en plus difficile de maintenir une dichotomie radicale entre le fonctionnement et l'histoire du vivant (Laland et al., 2011) [8]. C'est la raison pour laquelle certains chercheurs plaident en faveur d'une intégration de l'histoire évolutive dans la biologie fonctionnelle en articulant causes proximales et distales (Morange, 2003) [9]. Certains préconisent aussi une approche ontophylogénétique (Kupiec, 2012) [10] pour souligner l'importance entre le développement et l'évolution (évo-dévo) en particulier, en lien avec les modifications liées à la variation de l'expression des gènes au cours du développement, comme une nouvelle intelligibilité de la diversité temporelle du vivant.

La distinction entre biologie fonctionnaliste et biologie évolutive confère à la biologie une double nature lui faisant perdre, en apparence, son unité. S'il existe une légitimité épistémologique à distinguer le fonctionnement des organismes de leur histoire évolutive, les fonctions et les structures biologiques sont aussi le produit de cette histoire. Les explications causales du fonctionnement des organismes ne sont donc pas indépendantes de celles de la contingence historique. Le principe de contingence introduit par Gould (1991) [11] comme celui de la non-prédictivité d'événements singuliers aux cours des temps géologiques ne signifie pas que l'histoire évolutive ne soit pas connaissable ni explicable. L'absence de prédiction n'interdit pas une rétrodiction selon Gayon (1993) [12] c'est-à-dire l'identification rétrospectivement des causes matérielles qui ont présidées à des 
événements biologiques (spéciation, extinction, adaptation, etc.). Comprendre l'histoire du vivant reviendrait donc à comprendre le fonctionnement et l'organisation des espèces. La question de la causalité en biologie est donc plurielle, à la fois structo-fonctionnelle et historique (Forest et Malaterre, 2013) [13]. Certains chercheurs évoquent aussi un autre processus, non téléologique ni vitaliste: l'auto-détermination du vivant, pour expliquer l'émergence de structures fonctionnelles résultant du hasard et de la sélection (Cunchillos, 2014; Ricard, 2003) [14, 15]. Malgré le caractère polymorphe de la causalité en biologie de l'évolution, nul besoin de faire appel à un projet intelligent pour expliquer l'histoire du vivant (Brosseau et Silberstein, 2011) [16]. Les phénomènes évolutifs s'expliquent uniquement par des causes matérielles génétiques et/ou non génétiques. C'est pourquoi, l'introduction d'une diversification du vivant sans modification du génome dans le programme de la classe de T.S, présenté comme un complément d'informations sur les récentes avancées en biologie, interroge la dichotomie autant que la congruence entre causes proximales et distales, mais aussi la prise en compte dans le programme de la causalité évolutive au regard de la diversification du vivant.

\section{La causalité évolutive, une composante de la nature de la science}

Depuis quelques années, de nombreuses études mettent en avant l'importance d'un enseignement de la Nature de la Science (NoS) dans la formation scientifique des élèves. Pour Lederman (1992) [4], le cadre de référence de la NoS se décline en plusieurs points: la connaissance scientifique est provisoire, empiriquement fondée sur l'observation du monde naturel, elle a une part de subjectivité, elle engage l'imagination et la créativité, et est socialement et culturellement intégrée. Lederman (2007) [17] a réactualisé son cadre initial de la NoS pour souligner, entre autre, l'importance des constructions théoriques fonctionnelles et pas seulement des observations et données empiriques dans la connaissance scientifique. Le cadre initial de la NoS a aussi été élargi avec la prise en compte de l'interdisciplinarité (Erduran, 2014) [18] et de la dimension sociale de la science (Matthew, 2012) [19]. Si les travaux de Lederman privilégient l'unité des méthodes et des explications scientifiques, d'autres chercheurs défendent une approche en lien avec la spécificité disciplinaire, en particulier, pour la biologie de l'évolution en tant que science historique (Dagher et Boujaoude, 2005) [20]. À ce titre, les travaux en didactique des SVT menés par Orange-Ravachol (2010) [21] sur la problématisation du temps et son irréversibilité pointent du doigt la nécessité de dépasser le principe explicatif de l'actualisme pour intégrer le principe de contingence historique. D'autres travaux soulignent les limites d'un enseignement uniquement focalisé sur le contenu scientifique, en particulier, quand il s'agit de faire face à la controverse créationnisme versus évolutionnisme (Lombroso, Thanukos et Weisber, 2008; Pigliucci, 2007) [22, 23]. Ces travaux témoignent, à des degrés divers, de la difficulté qu'ont les élèves à conjuguer et à naviguer entre l'explication fonctionnaliste et l'explication historique de la diversification du vivant. Cette oscillation entre contingence historique et fonctionnement du vivant interroge sur ce qui est donné à voir, dans les programmes de SVT, de la nature de la science.

Dans le Préambule du programme de SVT de la classe de TS (MEN, 2011) [24], il est fait mention de la NoS comme d'un objectif d'enseignement. Plus précisément, il s'agit:

- d'aider à la construction d'une culture scientifique commune fondée sur des connaissances considérées comme valides tant qu'elles résistent à l'épreuve des faits (naturels ou expérimentaux) et des modes de raisonnement propres aux sciences (p. 1).

- de montrer (...) que la science construit, à partir de méthodes d'argumentation rigoureuses fondées sur l'observation du monde, une explication cohérente de son état, de son fonctionnement et de son histoire (p. 1).

\footnotetext{
${ }^{3}$ Préambule est commun à tous les programmes de SVT du lycée.
} 
- d'aider «à montrer la cohérence globale du champ intellectuel concerné, centré sur un objet d'étude - la nature - et des méthodes fondées sur la confrontation entre les idées scientifiques et les faits - naturels ou expérimentaux » (p. 1).

- que les élèves comprennent « la nature provisoire, en devenir, du savoir scientifique » et qu'ils pratiquent " une démarche scientifique (observer, questionner, formuler une hypothèse, expérimenter, raisonner avec rigueur, modéliser) (p. 5).

Concernant, plus particulièrement, la nature de la biologie de l'évolution qui donné à voir dans les programmes de SVT du secondaire, Orange (2009) [25] fait remarquer que les fonctions épistémologiques attribuées au concept d'évolution, tout au long du secondaire, sont le plus souvent limitées à la dimension factuelle et peu à la dimension de modèle explicatif ou de paradigme. Par exemple, la théorie de l'évolution n'est pas mentionnée dans les programmes, en classe de T.S, depuis des années 1990 jusqu'à aujourd'hui. Cette absence témoigne, sans doute, d'une difficulté à prendre en charge, d'un point de vue curriculaire, la question de la causalité évolutive (Fortin, 2009) [26] au vu de la pluralité des causes possibles de la diversification du vivant. L'introduction de la diversification sans modification du génome dans le programme de T.S permet d'interroger la nature de la biologie de l'évolution qui est donnée à voir aux élèves : celle d'une explication circonscrite ou bien celle d'une explication émergente quant à la diversification du vivant.

\section{Prescriptions et recommandations officielles}

Le Programme de SVT de la classe de TS est découpé en trois grands thèmes: La Terre dans l'Univers, la vie, l'évolution du vivant; Enjeux planétaires contemporains; Corps humain et santé. Le Thème 1 est décomposé en deux partie: 1.A «Génétique et évolution» et1.B «Le domaine continental et sa dynamique». L'item concernant la diversification du vivant sans modification du génome se situe dans le Thème 1-A, lui-même décliné en cinq sous-thèmes présentés dans le tableau ci-dessous:

Tableau 1. Présentation des sous-thèmes du Thème 1.A et connaissances associées.

\begin{tabular}{|l|l|}
\hline Sous-thèmes 1.A : Génétique et évolution & Connaissances clés pour chaque sous-thème \\
\hline $\begin{array}{l}\text { A1 : Le brassage génétique et sa contribution } \\
\text { à la diversité génétique }\end{array}$ & $\begin{array}{l}\text { Brassage intra et interchromosomique (méiose); } \\
\text { gamètes ; fécondation ; stabilité des caryotypes; } \\
\text { duplication de gènes et familles multigéniques; } \\
\text { anomalies chromosomiques ; hybridation; } \\
\text { polyploïdisation }\end{array}$ \\
\hline $\begin{array}{l}\text { A2 : Diversification génétique et } \\
\text { diversification des êtres vivants }\end{array}$ & $\begin{array}{l}\text { Variation de l'expression des gènes de développement ; } \\
\text { transfert de gènes par voie virale ; symbiose ; } \\
\text { acquisition de nouveaux comportements }\end{array}$ \\
\hline $\begin{array}{l}\text { A3 : De la diversification des êtres vivants à } \\
\text { l'évolution de la biodiversité }\end{array}$ & $\begin{array}{l}\text { Pression du milieu ; concurrence ; hasard ; sélection } \\
\text { naturelle ; dérive génétique ; spéciation ; population et } \\
\text { notion d'espèces }\end{array}$ \\
\hline $\begin{array}{l}\text { A4: « une regard » sur l'évolution de } \\
\text { l'Homme }\end{array}$ & $\begin{array}{l}\text { Ancêtre commun à l'Homme et au Chimpanzé ; } \\
\text { caractéristiques du genre Homo ; dynamique évolutive } \\
\text { des Primates ; mécanismes possibles à l'origine de la } \\
\text { diversification Homme / chimpanzé }\end{array}$ \\
\hline $\begin{array}{l}\text { A5 : Les relations entre organisation et mode } \\
\text { de vie, résultat de l'évolution : l'exemple de } \\
\text { la vie fixée chez les plantes }\end{array}$ & $\begin{array}{l}\text { Organisation fonctionnelle ; organisation et gènes de } \\
\text { développement de la fleur ; pollinisation ; fécondation ; } \\
\text { structures et mécanismes de défense ; co-écolution } \\
\text { fleurs/animaux }\end{array}$ \\
\hline
\end{tabular}


Nous nous intéresserons ici, à la «Diversification génétique et diversification des êtres vivants » (MEN, 2011; p. 7) [24]. Notons, d'emblée, qu'il n'est pas fait mention d'une diversification «non génétique» du vivant. Il existe dans l'énonciation de ce sous-thème, une tension entre la présence de la matérialité génétique et l'absence de modification du génome. Un petit texte d'accompagnement de l'intitulé apporte quelques précisions quant à la nécessité de recourir à d'autres explications:

«L'association des mutations et du brassage génétique au cours de la méiose et de la fécondation ne suffit pas à expliquer la totalité de la diversification génétique des êtres vivants. Il s'agit ici de donner une idée de l'existence de la diversité des processus impliqués, sans chercher une étude exhaustive. En outre, une diversification des êtres vivants n'est pas toujours liée à une diversification génétique. » (Ibid., p. 7) [24].

La dernière phrase donne acte au lecteur au professeur de faire cohabiter des explications de nature différentes. Concernant le dernier point, le Programme annonce une prescription de ce qui doit être enseigné. D'un côté, sont cités des mécanismes de diversification des génomes ou de variations de l'expression des gènes du développement, de l'autre côté les mécanismes de diversification sans modification du génome :

«D'autres mécanismes de diversification des génomes existent: hybridations suivies de polyploïdisation, transfert par voie virale, etc. S'agissant des gènes impliqués dans le développement, des formes vivantes très différentes peuvent résulter de variations dans la chronologie et l'intensité d'expression de gènes communs, plus que d'une différence génétique. 》

-Une diversification des êtres vivants est aussi possible sans modification des génomes : associations (dont symbioses) par exemple. Chez les vertébrés, le développement de comportements nouveaux, transmis d'une génération à l'autre par voie non génétique, est aussi source de diversité : chants d'oiseaux, utilisation d'outils, etc. » (Ibid., p. 7) [24].

Notre analyse porte sur le second item consacré à la diversification des êtres vivants sans modification du génome. Celui-ci n'est pas isolé des autres items. Il s'inscrit dans une mise en réseau des concepts du thème $1 \mathrm{~A}$ et doit être mis en perspective avec les programmes les classes précédents concernant les connaissances en génétiques ${ }^{4}$. Pour accompagner les enseignants dans la mise en œuvre du Programme, l'Inspection Générale de l'Education Nationale de SVT (IGEN de SVT) a produit un document "Ressources pour la classe Terminale Scientifique» (MEN, 2012) [27]. Concernant, l'item centré sur la diversification du vivant sans modification du génome, il est indiqué dans les Ressources que:

«D’une façon générale, à ce stade des connaissances, les élèves pensent que ce sont les mutations qui sont essentiellement à l'origine de la diversification des formes de vie, donc des génomes. Ils ont découvert lors de l'étude du thème 1-A-1 que de nouveaux gènes pouvaient apparaître par duplication puis modification (mutations) d'un gène préexistant. Il s'agit maintenant de leur faire prendre conscience: de la diversité des mécanismes de diversification des génomes, de l'existence et de l'importance des gènes impliqués dans le développement et des conséquences possibles de leurs variations, de la possibilité de diversification du vivant sans modifications génomes par mutations, de la possibilité de transmission de caractères comportementaux d'une génération à l'autre par voie non génétique » (Ibid. p. 7) [27].

Et il est précisé, « il s'agit ici d'aborder un domaine qui est relativement nouveau pour les élèves (et peut être aussi pour les enseignants) : la transmission de génération en génération de caractères qui ne résultent pas, du moins à l'origine, de l'expression du génome. » (Ibid. p. 9) [27].

\footnotetext{
${ }^{4}$ Notions de mutation, de sélection naturelle, de dérive génétique.
} 
Le document Ressources recommande de mettre l'accent «sur la pratique de la démarche d'investigation, sur l'argumentation scientifique, sur le développement de l'autonomie des élèves, dans une optique de formation scientifique et de compréhension de ce qu'est la science, en perpétuelle évolution en fonction des nouvelles données disponibles» (p. 7). Il s'agit d' "Aborder la diversification du vivant qui n'a pas pour cause initiale une modification du génome » (p. 9). C'est donc bien en termes de cause initiale qu'est posé le problème: cause initiale génétique ou non génétique de la diversification. Il est aussi préconisé « de mettre les élèves en activité autour d'étude de cas » (p. 9). L'objectif est de fournir des données fiables pour " permettre aux élèves d'argumenter sur le fait que certains caractères soient réellement transmis de génération en génération, bien que leur apparition ne semble pas résulter d'un mécanisme de diversification du génome » (p. 9). On note, ici, une certaine prudence dans la formulation « ne semble pas résulter ».

La mise en œuvre de ces recommandations s'appuient sur trois exemples: l'endosymbiose, le transfert horizontal de gènes, et le comportement animal.

Nous proposons, ci-dessous, quelques pistes de réflexion sur la dimension épistémologique et didactique de chacun de ces exemples en lien avec l'image de la NoS, et en particulier avec celle «d'une science en perpétuelle évolution en fonction des nouvelles données » (p. 7). Notons d'emblée que dans le Programme, l'item relatif à la diversification sans modification du génome cite deux exemples : celui de la symbiose et de la transmission de nouveaux comportements, alors que dans les Ressources, la symbiose est évoquée à partir de l'endosymbiose et du transfert horizontal de gènes. En quoi l'exemple des transferts horizontaux de gènes est-il pertinent pour illustrer, ici, la diversification des êtres vivants sans modification du génome ? Certes, il n'y a pas de mutation, de recombinaison, de duplication génétique, etc. mais le transfert de gènes s'apparente, cependant, à la production d'un organisme génétiquement modifié (OGM). De plus, le transfert de gène est aussi évoqué dans le Programme à propos de la diversification du génome : "D'autres mécanismes de diversification des génomes existent : hybridations suivies de polyploïdisation, transfert par voie virale, etc. » (MEN, 2011; p. 7) [24]. C'est qu'il s'agit, ici, de transfert horizontaux de gènes produit dans le cadre de l'endosymbiose.

\subsection{L'endosymbiose}

Les Ressources pointent du doigt l'intérêt de mettre les élèves en activité sur cet objet biologique singulier que sont les symbioses, « il s'agit d'aborder essentiellement des exemples d'endosymbioses, dans l'optique d'étudier l'origine probable et la transmission de cette association de génération en génération » (p. 9 Eduscol, DGESCO, IGEN) [27].

S'appuyer sur l'endosymbiose suppose une coopération à bénéfices réciproques entre les deux organismes, mais aussi une tolérance immunitaire interspécifique. Le Programme de la classe de T.S consacre plusieurs chapitres à l'immunité. Dans un de ces chapitres, un item précise que « Le système immunitaire, normalement, ne se déclenche pas contre des molécules de l'organisme ou de ses symbiotes » (MEN, 2011, p. 13) [24]. La coopération endosymbiotique ne renvoie pas seulement à une lecture fonctionnaliste, mais aussi à une lecture évolutionniste de l'immunité (Pradeu, 2009) [28] permettant le maintien de l'endosymbiose (Selosse, 2012) [29]. Sa pérennité existe dans le cadre d'un compromis évolutif par un gain de valeur sélective pour cette coopération dans le temps. Il semblerait que l'on puisse considérer le symbionte comme un exemple de co-construction de niche où l'endosymbiose résulterait d'une interaction entre une hérédité génétique entre partenaires et une hérédité non génétique écologique au sens définit par Danchin et al. (2011) [2]. Les Ressources mettent l'accent sur le fait que les endosymbiotes sont héritables. Ce qui suppose à la fois une cause proximale liée à hérédité génétique de chacune des deux espèces (ne serait-ce que du point de vue immunitaire) et une cause distale liée à une hérédité non génétique au sens d'une co-construction écologique dont la valeur sélective serait plus élevée que la valeur sélective de chacune des deux espèces isolées. Mais la référence à la valeur sélective n'est pas, explicitement, prise en compte. Sans 
doute ces exemples d'endosymbioses ont-ils, ici, une valeur préparatoire pour évoquer, ensuite, la survie des organismes face à la pression du milieu dans le sous-thème1.A3.

Un des objectifs du Programme vise à «de donner une idée de l'existence» de différents processus de diversification du vivant. Mais en l'absence de référence explicite à la théorie endosymbiotique (Margulis, 1970) [30], on peut se demander si le descriptif des mécanismes endosymbiotiques indépendamment ne risque pas de conduire à une vision rigidifiée, voire de chosifier les relations endosymbiotiques en privilégiant une mise en histoire, principalement, descriptive de la «capture» par les cellules eucaryotes de bactéries hétérotrophes et/ou autotrophes pour rendre compte de l'origine endosymbiotique des chloroplastes et/ou mitochondries. Cette approche fonctionnaliste de l'endosymbiose passe sous silence que l'origine endosymbiotique de ces deux organites interroge, nécessairement, l'origine phylogénique des eucaryotes à partir des procaryotes (Lake, 2009) [31]. La théorie endosymbiotique étayée par de nombreuses données phylogénétiques permet d'apporter un éclairage évolutif sur la diversification des cellules à partir d'une diversification sans modification initiale du génome.

\subsection{Le transfert horizontal de gène}

Les Ressources (MEN, 2012; p. 10) [27] citent deux exemples de transfert horizontaux : Les ascidies dont la paroi contient de la cellulose, et L'origine symbiotique des chloroplastes et des mitochondries. Comme précédemment, se joue, ici, une lecture fonctionnelle et/ou évolutive des modifications du vivant. Les transferts horizontaux de gènes lus dans un cadre fonctionnaliste apportent une nouvelle fonctionnalité aux organismes en bénéficiant. Mais, ils peuvent aussi être lus dans un cadre évolutionniste de diversification du vivant. En particulier, ils questionnent la représentation traditionnelle de l'arbre évolutif. L'histoire du vivant n'est plus uniquement semblable à celle d'une verticalité arbustive, mais elle se situe à la croisée de la verticalité phylogénique et de l'horizontalité des transferts de gènes entre espèces. Ainsi l'arbre est, maintenant, devenu un réseau inscrit dans la temporalité.

Endosymbiose/transfert horizontal de gènes ne sont pas, ici, dissociés. Ils renvoient aux causes proximales et distales de la diversification du vivant et posent, aussi, la question de l'identité biologique de l'espèce au vu de l'intégration de gènes d'autres espèces intégrés dans le génome. La notion d'hérédité est révisée. D'une vision traditionnelle par transmission génétique verticale des parents aux descendants, on passe à une vision par par flux de gènes entre espèces. Ces exemples sont présentés comme des modes de diversification du vivant où interagissent hérédité génétique et hérédité non génétique. Quant à l'image de la NoS, elle relève plutôt d'une science capable de décrire « la transmission de l'association de génération en génération » sans vraiment en discuter « l'origine probable » (MEN, 2012; p. 9) [27].

\subsection{La transmission non génétique de certains comportements}

Si les exemples précédents étaient centrés sur une dimension physio-écologique, l'exemple du comportement animal est résolument centré sur une dimension éthologique (Lorenz, 1957; Tinbergen, 1963) $[33,33]$ comme nous en informe l'extrait ci-dessous des Ressources (MEN, 2012; p. 9) [27] :

« il sera utile de préciser ce qu'est un comportement, les interactions qu'il comporte, et son importance dans des phases clés de la vie des êtres vivants (recherche de nourriture, rapprochement des partenaires pour la reproduction). On peut être amené à évoquer ici la notion "d'empreinte », processus d'apprentissage mis en jeu pendant le développement des jeunes et qui produit une modification durable d'un comportement. Cette empreinte pourrait être héritable, bien qu'elle ne soit pas génétique. Si cette notion est abordée, elle doit l'être dans un contexte où la sélection naturelle est abordée, car cette héritabilité ne se fera que si l'empreinte affecte la valeur sélective. Certains exemples (concernant notamment la diversification de 
populations d'oiseaux (pouillot véloce/pouillot ibérique) en fonction du chant sont des supports intéressants pour travailler la démarche expérimentale, démarche suivie par les chercheurs dans ce domaine (on parle de « démarche éthologique »). En effet, les chercheurs enregistrent les différents chants ou cris, les comparent pour repérer des séquences qui diffèrent selon les populations, font des hypothèses sur l'implication de ces modifications dans la « séparation des populations ", puis testent leurs hypothèses en créant des séquences modifiées et en observant leur effet sur les populations naturelles. » (p. 9 Eduscol SVT, DGESCO, IGEN, 2012) [27].

Cet extrait fait référence à des notions complexes de comportement, d'empreinte, d'héritabilité, de valeur sélective, et de démarche éthologique. Les exemples cités sont les suivants :

«Pour la transmission de comportement à l'origine de la diversification des populations : Chants d'oiseaux, notamment chez les oiseaux-chanteurs ou oiseaux oscines (des études ont été réalisées chez la Paruline à sourcils blancs ou encore chez le Diamant mandarin), Pouillot véloce et pouillot ibérique. Utilisation d'outils chez certains primates (notion de « culture » chez les chimpanzés par exemple)». (Ibid., p. 10) [27]

\subsubsection{Le chant des oiseaux}

Les facteurs qui déclenchent la production du chant sont, généralement, des signaux visuels et/ou auditifs, mais aussi hormonaux que l'on peut qualifier de causes proximales. Il existe aussi des causes distales qui augmentent la fitness de l'espèce, en particulier, quand le chant est associé à la défense territoriale ou à la reproduction. Des expériences d'isolement des oisillons (Diamant mandarin) ont permis de montrer que les jeunes oiseaux doivent apprendre d'un tuteur (Catchpole et Slater, 1995) [34] en reproduisant son chant en fonction du contexte (empreinte vocale). D'autres études, chez la Paruline à sourcils blancs, ont mis en évidence que l'identification du partenaire se fait sur quelques notes. L'exemple du Pouillot véloce et du Pouillot ibérique montre un phénomène d'isolement éthologique par production de chants différents entre deux espèces morphologiquement identiques (espèces jumelles). Ainsi, parmi les oiseaux-chanteurs, les populations qui présentent des variantes dans leurs chants (dialectes) peuvent être isolées, d'un point de vue éthologique, même si elles partagent un même territoire. Dans ce cas, ces populations finissent par ne plus se reconnaître, faute d'un dialecte sonore commun, et un processus de spéciation peut être engagé.

\subsubsection{L'utilisation des outils chez les primates}

Les premiers travaux scientifiquement documentés ont décrit l'utilisation d'outils chez le chimpanzé pour casser des noix (Boesch et Boesch, 1983) [35] ou en ramassant des fourmis et des termites à l'aide d'une tige préalablement effeuillée (Mac Grew, 1987) [36]. Des travaux plus récents font apparaître l'existence de différentes pratiques d'utilisation d'outils entre communautés de chimpanzés qui partagent le même environnement écologique et la même nourriture (Luncz, Mundry et Boesch, 2012; Rawlings, Davila-Ross et Boysen, 2014) [37, 38]. Ainsi, les chimpanzés sélectionnent, utilisent, voire produisent des outils pour se nourrir, mais ils assurent aussi une transmission intergénérationnelle par le biais d'un long apprentissage de différentes techniques au sein de chacune des communautés. Sommes-nous en présence d'une culture non humaine ? Les Ressources évoquent la notion de «culture » chez les primates non humains sans préciser ce qu'il faut en dire aux élèves. Faut-il discuter, avec les élèves, de cette notion de culture et la proposer comme une donnée de réflexion sur les rapports entre les autres grands singes et l'humain dans le cadre du débat sur la notion de cultures non humaines (Laland et Hoppitt, 2003) [39]? La question reste ouverte puisqu'aucune indication n'est donnée dans les textes officiels. Ce débat sur une culture non humaine n'est pas sans rappeler un débat né dans les années 1960 et toujours d'actualité, à savoir faut-il classer le chimpanzé dans le genre Homo ? Les travaux de l'équipe de Goodman (Wildman et al., 2003) [40] consacrés à l'étude de séquences de différents gènes chez l'Homme et le Chimpanzé ont révélé qu'il 
existait une divergence moyenne entre les deux espèces de $1,14 \%$. Sur la base de ce résultat, et sachant que l'Homme et le Chimpanzé sont phylogénétiquement des groupes-frères issus d'un ancêtre commun exclusif, Goodman et son équipe ont proposé de les réunir dans un seul et même genre Homo. Statuer sur la frontière qui sépare culturellement l'humain des autres primates interroge la parenté biologique en termes de continuité et de rupture sans que l'on puisse, pour le moment, y apporter de réponse définitive. On retrouve, dans cet exemple, la question de l'articulation entre les causes proximales avec l'émergence de la culture et les causes distales avec la culture comme facteur de diversification entre communautés de chimpanzés. L'héritabilité du chant ou de l'utilisation d'outils conjugue d'une part, une origine génétique dans la capacité à chanter ou à prendre en main des d'objets (causes proximales) et d'autre part, l'impact évolutif de l'apprentissage individuel et collectif des comportements au sein des populations (causes distales).

Ces exemples montrent que l'acquisition d'un comportement n'est pas uniquement déterminé par les gènes mais qu'il dépend aussi de l'apprentissage c'est-à-dire d'une transmission culturelle. Le Programme et les Ressources mettent l'accent sur la valeur sélective du comportement du chant des oiseaux qui contribue à la survie des individus et des populations. Pour certains auteurs, il existerait une évolution culturelle résultant d'une sélection des variants comportementaux (Danchin et al., 2004) [41]. L'hérédité culturelle suppose que les jeunes n'apprennent pas nécessairement de leurs parents, mais aussi d'autres membres non apparentés. Cependant, ni dans le Programme ni dans les Ressources, le concept d'héritabilité n'est défini ou explicité. L'image de la NoS est, ici, celle d'une science d'une description de l'héritabilité des comportements située au regard de la valeur sélective des certains d'entre eux (exemple : chant chez les oiseaux).

\section{Conclusion}

De l'analyse des prescriptions et recommandations officielles, il apparaît une cohabitation entre causes non génétiques et causes génétiques de la diversification du vivant. Il n'en demeure pas mois que l'hérédité non génétique interroge les limites actuelles de la théorie de l'évolution, et ce faisant pointe le caractère provisoire de connaissances scientifiquement étayées. L'image de la NoS qui en ressort est celle d'une science construite sur un corpus constitué par l'accumulation d'éléments fonctionnels (voire factuels) mettant en avant les causes proximales (exemple : description de l'endosymbiose, de la transmission de comportements) et peu celle d'une science qui interroge en termes de causes distales la diversification du vivant.

Ce choix s'explique, peut-être, par un élément du Préambule (Danchin et al., 2011 ; p. 2) [2] : « Si les connaissances scientifiques à mémoriser sont raisonnables, c'est pour permettre aux enseignants de consacrer du temps pour faire comprendre ce qu'est le savoir scientifique, son mode de construction et son évolution au cours de l'histoire des sciences ». Les Ressources tout en reconnaissant que la diversification non génétique est un domaine nouveau pour les élèves (mais aussi parfois pour les enseignants) ne donnent aucune pistes concrète sur les attendus relatifs à une construction historique. Certes, l'enseignant(e) peut toujours prendre en charge le questionnement des élèves sur les causes non génétiques de la diversification du vivant. Mais il (elle) ne peut porter seul(e) la responsabilité d'une transposition didactique de savoirs scientifiques émergents. Comme le soulignent Maurines et al. (2013) [42], la présentation de NoS dans le Préambule du Programme n'est pas associée à un contenu d'enseignement précis, ce qui peut conduire les enseignants à ne pas s'attarder sur les caractéristiques de la NoS faute de pouvoir les identifier (Abd-El-Khalick et Lederman, 2000) [43]. Aussi, la question de la prise en charge curriculaire de la nature de la biologie de l'évolution est à mettre en regard des causes matérielles, à l'origine de la diversification, qui ne soient pas strictement d'ordre génétique. Une des pistes possibles d'une prise en charge curriculaire pourrait être d'interroger la diversification dans sa composante fonctionnelle et évolutive en lien avec une référence explicite à un cadre théorique donné. 
La « vie » et le « vivant » : de nouveaux défis à relever dans l'éducation

\section{Références}

1. H. Atlan, La fin du tout génétique? Vers de nouveaux paradigmes en biologie (INRA Editions, Paris, 1999).

2. É. Danchin, A. Charmantier, F. A. Champagne, A. Mesoudi, B. Pujol, S. Blanchet, Beyond DNA: integrating inclusive inheritance into an extended theory of evolution. Nature Reviews Genetics, 12, 475-486 (2011).

3. M. Pigliucci, G. B. Müller, Evolution: The Extended Synthesis (MIT Press, Cambridge, MA, 2010).

4. N. Lederman, Students' and teachers' conceptions of the nature of science: a review of the research, Journal of research in Science Teaching, 29, 4, 331-360 (1992).

5. E. Mayr, Cause and effect in Biology, Science, 134, 3489, 1501-1506 (1961).

6. A. A. Agrawal, Phenotypic Plasticity in the Interactions and Evolution of Species, Science, 294, 321-326 (2001).

7. T. Piersmai, J.A Van Gils, The flexible phenotype: a body-centred integration of ecology, physiology, and behaviour (Oxford University Press, Oxford, 2011).

8. K. N. Laland, K. Sterelny, F.J. Odling-Smee, W. Hoppit, T. Uller, Cause and effect in biology revisited: is Mayr's proximate-ultimate dichotomy still useful? Science. 334, 1512-1516 (2011).

9. M. Morange, Biologie : Une causalité éclatée, dans L. Viennot et C. Debru, Enquête sur le concept de causalité (p. 161-177) (PUF, Paris, 2003).

10. J. J. Kupiec, L'ontophylogenèse. Évolution des espèces et développement de l'individu (Quae, Versailles, 2012).

11. S.J. Gould, La vie est belle (Seuil, Paris, 1991).

12. J. Gayon, La biologie entre loi et histoire, Philosophie, Minuit, 38, 50-57 (1993).

13. D. Forest, C. Malaterre, La causalité en biologie, dans J. Gayon, T. Pradeu, Philosophie de la biologie, (p. 61-77) (Textes clés, Vrin, Paris, 2013).

14. C. Cunchillos, Les voies de l'émergence. Introduction à la théorie des unités de niveau d'intégration (Belin, Paris, 2014).

15. J. Ricard, Emergence, organisation et causalité dans les systèmes biologiques, dans L. Viennot et C. Debru, Enquête sur le concept de causalité (p. 179-195) (PUF, Paris, 2003).

16. O. Brosseau, M. Silberstein. Évolutionnisme(s) et créationnisme(s) dans T. Heams, P. Huneman, G. Lecointre, M. Silberstein (dir.), Les mondes darwiniens. (p. 1587_1512) (Éditions Matériologiques, Paris, 2011).

17. N.G. Lederman, Nature of science: Past, present, and future, in S. K. Abell \& N. G. Lederman (Eds.), Handbook of research on science education (p. 831-880) (Lawrence Erlbaum Associates, Mahwah, NJ, 2007).

18. S. Erduran, Beyond nature of science: The case for reconceptualizing « science » for science education, Science, Education International, 25, 1, 93-111 (2014).

19. M.R. Matthew, Changing the focus: from Nature of Science (NoS) to Features of Science (FoS), in M.S. Khine (Ed.), Advances in Nature of Science Research. Concepts and Methodologies (p. 3-26) (Springer, Dordrecht, 2012).

20. Z.R Dagher, S. Boujaoude, Students' perceptions of the nature of evolutionary theory, Science Education, 89, 378-391 (2005).

21. D. Orange-Ravachol, Problématisations fonctionnaliste et historique dans la construction de savoirs et les apprentissages en sciences de la Terre et de la vie : entre continuité phénoménale et discontinuité événementielle, Mémoire d'habilitation à diriger des recherches, Université de Nantes (2010).

22. T. Lombroso, A. Thanukos, M. Weisberg, The importance of understanding the nature of science for accepting evolution, Evolution: Education and Outreach, 1, 290-298 (2008).

23. M. P. Pigliucci, The evolution-creation wars: why teaching more science is just not enough, McGill Journal of Education, 42, 285-306 (2007).

24. Ministère de l'Éducation Nationale, Bulletin officiel spécial n ${ }^{\circ} 8$ du 13 octobre 2011. 
25. C. Orange, Les fonctions épistémologiques de l'évolution dans les programmes français de sciences de la vie et de la Terre, dans M. Coquidé et S. Tirard (dir.), Évolution du vivant : un enseignement à risque? (p. 35-43) (Vuibert Adapt, Paris, 2009).

26. C. Fortin, L'enseignement de l'évolution au lycée, entre description et explication, dans M. Coquidé et S. Tirard (dir.), L'évolution du vivant : Un enseignement à risque? (p. 15-34) (Vuibert, Paris, 2009).

27. Inspection Générale de l'Education Nationale de Sciences de la vie et de la Terre, Eduscol, MEN, IGEN SVT (2012).

28. T. Pradeu, Les Limites du soi. Immunologie et identité biologique (Fides, Montréal/Vrin, Paris, 2009).

29. M. A. Selosse, Symbiose et mutualisme versus évolution : de la guerre à la paix ? Atala, 15, 3549 (2012).

30. L. Margulis, Origin of Eukaryotic Cells (Yale University Press, New Haven, 1970).

31. J.A. Lake, Evidence for an early prokaryotic endosymbiosis, Nature, 460, 967-971 (2009).

32. K. Lorenz, The companion in bird's world, In C.H. Schiller C.H. (ed.), Instinctive Behavior, (p. 83-128) (International Universities Press, New York, 1957).

33. N. Tinbergen, On aims and methods of Ethology, Zeitschrift für Tierpsychologie, 20, 410-433 (1963).

34. C. K. Catchpole, P. J. B. Slater, Bird song, biological themes and variations (Cambridge University Press, Cambridge, 1995).

35. C. Boesch, H. Boesch, Optimisation of nuts-cracking with natural hammers by wild chimpanzees, Behavior, 83, 265-286 (1983).

36. W. Mac Grew, Tools to get food: the subsitants of Tasmanian Arborigines and Tanzanian chimpanzees comparared, Behavior, 43(3), 247-258 (1987).

37. L. V. Luncz, R. Mundry, C. Boesch, Evidence for Cultural Differences between Neighboring Chimpanzee Communities, Current Biology, 22(10), 922-926 (2012).

38. B. Rawlings, M. Davila-Ross, S. Boysen. Semi-wild chimpanzees open hard-shelled fruits differently across communities. Animal Cognition, 17(4): 891. (2014).

39. K. N. Laland, W. Hoppitt, Do animals have culture? Evol. Anthropol, 12, 150-159 (2003).

40. D. E. Wildman, M. Uddin, G. Liu, L. Grossman, M. Goodman, Implications of natural selection in shaping $99.4 \%$ nonsynonymous DNA identity between humans and chimpanzees: Enlarging genus Homo, Current Issue, 100(12) (2003).

41. É. Danchin, L.A. Giraldeau, T. Valone, R. Wagner. From nosy neighbours to cultural evolution, Science, 305, 487-491 (2004).

42. L. Maurines, M. Gallezot, M.J. Ramage, D. Beaufils, La nature des sciences les programmes de seconde de physique-chimie et de sciences de la vie et de la Terre, Recherche en didactique des sciences et des technologie, 7, 19-52 (2013).

43. F. Abd-El-Khalick, N.G. Lederman, The influence of History of Science Courses on Students' views of Nature of Science, Journal of research in science teaching, 37(10), 1057-1095 (2000). 